\title{
Los niños con necesidades especiales y su seguridad como ocupantes de automóviles
}

Publicado en Internet: 21-marzo-2013

Jesús Monclús González: jmonclus@movilidad.org

\author{
J. Monclús González ${ }^{\mathrm{a}}$, A. Galdón ${ }^{\mathrm{b}}$

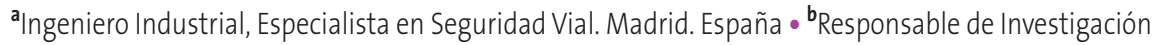 \\ del Instituto de Seguridad Vial de la Fundación MAPFRE. Madrid. España.
}

Palabras clave:

- Necesidades especiales

- Ocupantes

de automóviles

- Accidentes de tráfico
Objetivo: el objetivo de este artículo es abrir un debate en España sobre la seguridad en el automóvil de los niños con necesidades especiales.

Material y métodos: se han revisado y resumido los principales manuales y guías sobre la materia existentes en EE. UU., Canadá y Suecia. Se han tabulado las soluciones técnicas recomendadas para alrededor de 30 condiciones o necesidades especiales. Se ha realizado un análisis de mercado inicial, vía e-mail, de soluciones técnicas disponibles en España.

Resultados: se estima que en España hay al menos 60000 niños con necesidades especiales desde el punto de vista de su transporte seguro en el automóvil. Son varios los países que ofrecen manuales o guías sobre la materia: EE. UU., Canadá y Suecia. Este no es el caso de España. Mientras que algunos niños con necesidades especiales pueden utilizar asientos infantiles convencionales, otros necesitan soluciones técnicas especializadas. No todas están disponibles en España; de hecho, el mercado nacional es muy limitado. Los asientos especiales pueden ser caros y necesitarse durante cortos periodos de tiempo.

Conclusiones: se espera que el presente resumen sirva como punto de partida para un debate posterior, liderado por la comunidad pediátrica española, sobre el transporte seguro en el automóvil de niños con necesidades especiales. El debate debería finalizar con la redacción y difusión de una guía para profesionales médicos, padres y tutores (existen ya ejemplos a nivel internacional). Sería recomendable la promoción de programas de ayuda económica y préstamos de asientos especiales para determinadas condiciones médicas especiales.

\section{The safety of children with special needs as car occupants}

Objective: to open a debate in Spain about the safety of children with special needs as car passengers.

Material and methods: a review of the international literature has been undertaken in order to identify related articles, handbooks and guides. Several ones have been found in EE.UU., Canada and Sweden. Findings have been summarized in a table with technical solutions for around 30 different medical conditions. A preliminary market analysis in Spain has been performed.

Results: the number of children with special needs from the point of view of their safety in the automobile has been estimated in Spain in around 60,000. Several countries have developed handbooks or guides on this matter. This is not the case in Spain. Some children with special needs can use conventional child safety seats; others need specialized (medical) seats. Not all technical solutions available in other countries are sold in Spain; the number of solutions in the Spanish market, in fact, is very small. Special needs seats are normally very expensive; in some cases they are only needed during a short period of time.

Conclusions: the authors aim to trigger a more profound debate in Spain about the safety of special needs children as car passengers. This debate should be steered by the Spanish pediatrician community. The final outcome should be a handbook for medicine practitioners, parents and tutors (several

Key words:

- Special needs

- Car occupants

- Traffic crashes examples exist at international level). It is recommended to implement purchase and loans programs to support the use of specialized child seats. 


\section{INTRODUCCIÓN}

Los accidentes de tráfico siguen constituyendo en España la principal causa externa de muerte infantil, representando en el año 2010, último año para el que se dispone de esta información en la base de datos del Instituto Nacional de Estadística (INE, www.ine.es), un $37 \%$ de todas las muertes de niños de 0 a 14 años sucedidas en España y repuntando desde el 34\% correspondiente al año 2009. Los ahogamientos, con un $27 \%$ y las caídas accidentales, con un $8 \%$, fueron las otras dos causas externas más frecuentes de muerte infantil (niños de 0 a 14 años) en España y en el año 2010

En 2010, un total de 79 niños de entre 0 y 14 años murieron en España como consecuencia de siniestros de tráfico. La suma de niños fallecidos y heridos graves en 2010 ascendió a 489. En el año 2009, el número de niños fallecidos en España como consecuencia de accidentes de tráfico fue de 60: entre los años 2009 y 2010, por tanto, se produjo un importante aumento, del $32 \%$, en el número de niños fallecidos en España como consecuencia de accidentes de tráfico ${ }^{1}$.

La seguridad de los niños en el automóvil ha recibido una notable atención en los últimos años. Fruto de ello, su siniestralidad se ha visto reducida un $80 \%$ en los últimos 20 años $^{1}$. Así, por ejemplo, el uso general de sistemas de retención infantil ha aumentado progresivamente hasta alcanzar cifras cercanas al $90 \%$ en la actualidad ${ }^{2}$. Los asientos infantiles de seguridad para el automóvil previenen entre el 50 y el $90 \%$ de todas las lesiones graves o mortales ${ }^{3}$. Sin embargo, y sin dejar el campo de la seguridad vial infantil, existe un área en la que, muy probablemente, se ha avanzado mucho menos: la seguridad en el automóvil de los niños con necesidades especiales.

Un ejemplo de ello puede ser el siguiente testimonio reciente sobre la problemática a la que se enfrentan los padres con niños con necesidades especiales: "Tengo un niño de 15 meses y $11 \mathrm{~kg}$ de peso con un retraso psicomotor grave, debido a su enfermedad neurológica (tiene una lisencefalia y encefalopatía epiléptica) todavía no tiene control cefálico y presenta una hipotonía generalizada; he leído algunos documentos donde recomiendan sentar al niño en sentido contrario a la marcha, aunque sigo echando en falta una lista de las distintas sillas de auto o dispositivos existentes en el mercado que se puedan utilizar en este tipo de casos".

Resulta obvio que los niños con necesidades especiales deberían beneficiarse del mismo nivel de seguridad que el resto de niños. De hecho, en realidad, muchos niños con necesidades especiales deberían contar con un nivel adicional de seguridad, ya que su cuerpo es mucho más sensible o frágil que el de un niño normal. Por ello, e independientemente de que las condiciones médicas sean temporales o permanentes, los niños con necesidades especiales deberían viajar siempre en sistemas de retención infantil adecuados para sus condiciones particulares. La pregunta siguiente es: ¿Cuáles son, entonces, dichos sistemas de retención adecuados?

El objetivo de este artículo es resumir un reciente estudio sobre la temática elaborado por el Instituto de Seguridad Vial de la Fundación MAPFRE ${ }^{4}$. En ambos casos, en el de este artículo y en el del citado estudio, el objetivo perseguido es poner a disposición de padres y profesionales de la salud información y referencias internacionales relacionadas con el transporte en vehículos particulares de niños con necesidades especiales. Queda fuera del alcance de este trabajo pretender ofrecer consejo técnico específico, puesto que este, en todo caso, debería ser fruto de un debate posterior. Tampoco es objeto de este trabajo proponer cambios en la legislación vigente sobre asientos infantiles para el automóvil. En el medio plazo, por otra parte, el objetivo de esta línea de trabajo es ayudar a que las familias con niños con necesidades especiales, quienes se enfrentan diariamente a un elevadísimo número de retos de gran calado, cuenten al menos en el ámbito de la seguridad en el automóvil con consejos y soluciones técnicas de ayuda para sus niños.

Para finalizar esta introducción, conviene indicar que el artículo ha sido preparado por autores cuyo 
bagaje no es médico, sino técnico, por lo que probablemente existan imprecisiones que, en cualquier caso, no deberían afectar a la validez de los resultados y conclusiones de este trabajo.

\section{MATERIAL Y MÉTODOS}

En el marco de este trabajo se ha revisado la bibliografía internacional relacionada en busca de referencias, consejos o soluciones técnicas que ayuden a que los niños con necesidades especiales se beneficien del mismo nivel de seguridad, cuando viajan en el automóvil, que el resto de niños. A nivel internacional, se han encontrado referencias sobre el transporte seguro por carretera de los niños con necesidades especiales principalmente en EE. UU., Canadá y Suecia. Las fuentes básicas de información identificadas han sido:

- Documentos elaborados por la Academia Americana de Pediatría 5,6

- Guía elaborada por el Ministerio de Transportes de Canadá7.

- Manual sueco "Viajar seguro-Seguridad vial para niños con discapacidad"8.

- Programa del Hospital Infantil Riley de la Universidad de Indiana (www.preventinjury.org, página web consultada el 13 de enero de 2013).

- Manual Goodwill sobre seguridad en el automóvil y niños con necesidades especiales ${ }^{9}$.

- Boletín monográfico sobre la materia elaborado por Safe Ride New ${ }^{10}$.

La información recabada a nivel internacional se ha completado con una serie de entrevistas o contactos puntuales con actores clave españoles e internacionales (profesionales médicos, ortopedias, fabricantes de asientos infantiles, asociaciones de personas con necesidades especiales...).

\section{RESULTADOS}

\section{Quiénes y cuántos son}

En el ámbito que nos ocupa, los niños con necesidades especiales son todos aquellos niños con al- gún tipo de condición médica particular que exija unos cuidados o una atención especial a la hora de su transporte. Entre dichas condiciones se incluyen, por ejemplo, los niños prematuros o con bajo peso al nacer, los niños con espina bífida, con parálisis cerebral, con enfermedades neuromusculares o con falta de control muscular, con huesos de cristal, con autismo o con otros desórdenes de comportamiento, etc.

Uno de los anexos del estudio en el que está basado este artículo describe brevemente, y para el lector no experto, las necesidades especiales más frecuentes $y$, cuando se ha dispuesto de dicho dato, se incluye también el dato de la prevalencia o la tasa de niños con cada una de las diferentes condiciones médicas ${ }^{4}$. El resumen de la incidencia poblacional de cada una de las enfermedades o condiciones médicas para las que se ha encontrado un dato concreto sobre su incidencia se ofrece en la Tabla 1.

Con los datos anteriores -y bajo la hipótesis de que ciertas condiciones como la displasia de cadera, el síndrome de Asperger, el déficit de atención o la hiperactividad son tratables o curables y solo afectan a los niños durante, como media conservadora, un año de vida (por ejemplo, durante el primer año tras el nacimiento)-, se puede concluir que el número de niños con necesidades especiales en España se sitúa, muy probablemente, en los 60000 niños (en concreto, 59500 niños).

El cálculo anterior ha tenido en cuenta que, en España, nacen cada año alrededor de 400000 niños, y la población infantil de entre 0 y 14 prácticamente alcanza los 7000 000. Puesto que en este cálculo no se han incluido todas las necesidades o condiciones especiales identificadas, la incidencia global en la población española es muy probablemente superior a esta cifra.

Por otro lado, una revisión sueca de la bibliografía sobre niños con necesidades especiales indica que cada año nacen en Suecia aproximadamente 100000 niños y que más del 1\%, probablemente entre el 2 y el 5\%, sufre algún tipo de discapacidad durante la infancia ${ }^{11}$. Otro estudio estadounidense que analizó datos recopilados entre 1998 y 2002 


\begin{tabular}{|l|l|}
\hline \multicolumn{1}{|c|}{ Tabla 1. Incidencia estimada de determinadas condiciones médicas } \\
\hline Síndrome de Down & Uno de cada 800 nacimientos \\
\hline Acondroplasia & Uno de cada 25000 niños \\
\hline Atrofia muscular espinal & $\begin{array}{l}\text { En España nacen unos } 100 \text { casos nuevos por año y se considera que actualmente hay } \\
\text { más de } 1500 \text { familias con pacientes afectados }\end{array}$ \\
\hline $\begin{array}{l}\text { Déficit de atención con } \\
\text { hiperactividad }\end{array}$ & $\begin{array}{l}\text { Se estima que lo padece el } 5 \% \text { de la población infantojuvenil, lo que equivale a uno o } \\
\text { dos niños por aula }\end{array}$ \\
\hline Displasia de cadera & Aparece en aproximadamente tres de cada 1000 recién nacidos \\
\hline Distrofia muscular & $\begin{array}{l}\text { Se considera que la distrofia muscular en general tiene una incidencia cercana a uno } \\
\text { entre cada } 2000 \text { nacimientos }\end{array}$ \\
\hline Espina bífida (mielomeningocele) & $\begin{array}{l}\text { La incidencia estimada de espina bífida fue de 2,87 afectados por cada } 10 \text { 000 bebés } \\
\text { nacidos vivos en } 1996\end{array}$ \\
\hline Fibrosis quística & $\begin{array}{l}\text { Se estima que la incidencia de la fibrosis quística en España es de un caso por cada } \\
3500 \text { bebés nacidos vivos }\end{array}$ \\
\hline Osteogénesis imperfecta (OI) & En España podría haber un mínimo de 2700 afectados por alguno de los tipos de OI \\
\hline Parálisis cerebral & $\begin{array}{l}\text { Se presenta en dos de cada } 1000 \text { nacidos vivos. En España, alrededor de } 1500 \text { bebés } \\
\text { nacen con una parálisis cerebral o la desarrollan cada año }\end{array}$ \\
\hline Síndrome de Asperger & $\begin{array}{l}\text { No hay estudios que reflejen unas cifras exactas, pero se considera que el síndrome de } \\
\text { Asperger afecta a entre tres y siete niños por cada } 1000 \text { personas }\end{array}$ \\
\hline
\end{tabular}

sobre niños menores de 17 años implicados en accidentes de circulación (en total se recopilaron datos relativos a 14654 niños de edades comprendidas entre los 4 y los 15 años) concluyó que, de los niños incluidos en el estudio, un total de 152 niños (el 1,1\% aproximadamente) tenían algún tipo de necesidad especial relacionada con la conducta: déficit de atención y desorden de hiperactividad, discapacidades intelectuales, etc. Teniendo en cuenta, como se ha indicado, que según datos del INE, la población española de niños de entre 0 y 14 años es de 6936340 niños y aplicando el citado porcentaje mínimo de niños con discapacidad del 1\%, se concluye que en España existirían un mínimo de 69500 niños con discapacidad. Esta cifra coincide grosso modo con la calculada más arriba a partir de la incidencia de determinadas enfermedades o condiciones médicas.

En la bibliografía internacional se citan cerca de 30 condiciones médicas o necesidades especiales. Las condiciones particulares y las necesidades especiales para las que la revisión de la bibliografía internacional ofrece algún tipo de sugerencia, recomendación o solución técnica son:

- Bebés prematuros o con bajo peso al nacer.
- Niños con problemas respiratorios.

- Niños que necesariamente deban viajar tumbados.

- Niños con secuencia de Pierre Robin.

- Niños que padecen reflujo.

- Niños con escaso control a nivel de cuello o cabeza.

- Niños con parálisis cerebral.

- Niños con enfermedades musculares.

- Niños con espina bífida u otras lesiones medulares espinales.

- Niños con escoliosis.

- Niños con hidrocefalia.

- Niños con osteogénesis imperfecta ("niños de cristal").

- Niños con reumatismo.

- Niños con acondroplasia (enanismo).

- Niños grandes u obesos.

- Niños que usan gafas.

- Niños con problemas de visión.

- Niños con problemas auditivos.

- Niños con vendajes.

- Niños con collarines 
- Niños con traqueotomías.

- Niños con tubos de alimentación o gastrointestinales.

- Niños con yesos.

- Niños portadores de halo craneal.

- Niños con comportamientos difíciles.

- Niños con retrasos en el desarrollo.

- Niños que se desplazan en sillas de ruedas.

- Niños que deben viajar con equipo médico.

\section{Datos hallados en la revisión bibliográfica}

Uno de los primeros resultados que se han obtenido de la revisión de la bibliografía es que, a menudo, los niños con necesidades especiales pueden utilizar asientos convencionales (aquellos a la venta en establecimientos, homologados según la normativa técnica general para sistemas de retención infantil y que no han sido diseñados pensando específicamente en niños con necesidades especiales) pero, en otras ocasiones, resulta conveniente o imprescindible usar asientos especiales o adaptados a las condiciones particulares de cada niño. Normalmente, los asientos especiales o adaptados son más difíciles de adquirir y, en todos los casos, resultan notablemente más caros, con precios que pueden superar ampliamente los 1000 euros.

En las Figs. 1 a 5 se muestran algunos ejemplos de soluciones específicas para niños con determinadas condiciones o necesidades especiales que, o bien no están disponibles en España, o bien no son fáciles de encontrar en el mercado nacional.

Figura 1. Propuesta de arnés para transporte en posición supina de niños con yesos ${ }^{6}$

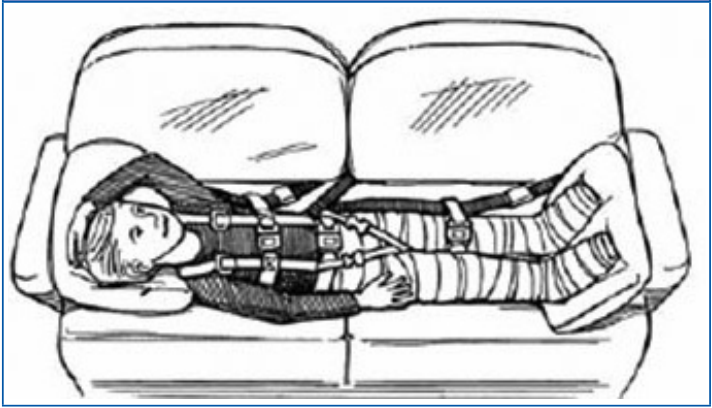

Figura 2. Silla Britax Hippo, sin laterales inferiores para acomodar yesos de espica

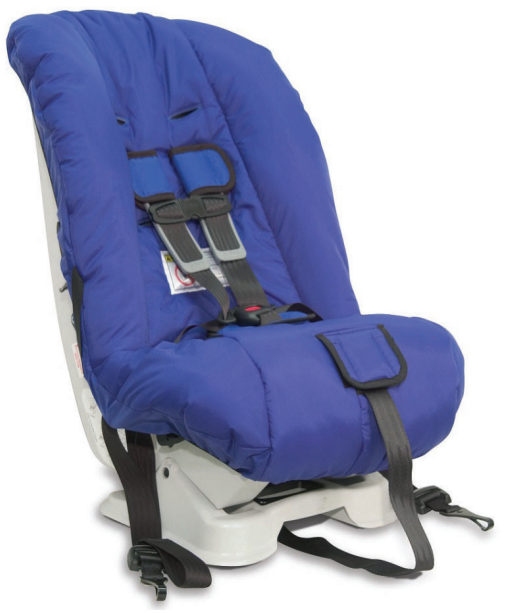

A continuación se resume en forma de tabla, para cada tipo de asiento o sistema de retención o movilidad, cuáles son las diferentes necesidades o condiciones especiales para las que está explícitamente indicado. La Tabla 2a muestra la relación entre los sistemas de retención infantil convencionales y las distintas condiciones médicas, mientras que la Tabla 2 b muestra la relación entre aquellos sistemas de retención especialmente diseñados para niños con necesidades especiales y dichas necesidades. En las tablas se utilizan las siguientes abreviaturas:

- REC: sistema de retención o movilidad RECOmendado o considerado como opción preferida.

- POS: sistema de retención que es POSible que pueda ser utilizado por algunos de los niños con las necesidades especiales indicadas, en función de la afección concreta, del grado de esta, y de las recomendaciones específicas de su pediatra o terapeuta. En otras palabras, se trata de un sistema de retención sin contraindicaciones generales para la condición o necesidad especial en cuestión.

- ESP: sistema de retención para casos ESPeciales en los que no sea posible el uso de un sistema de retención convencional. 
Figura 3. Asientos especializados para niños mayores que necesitan apoyo postural

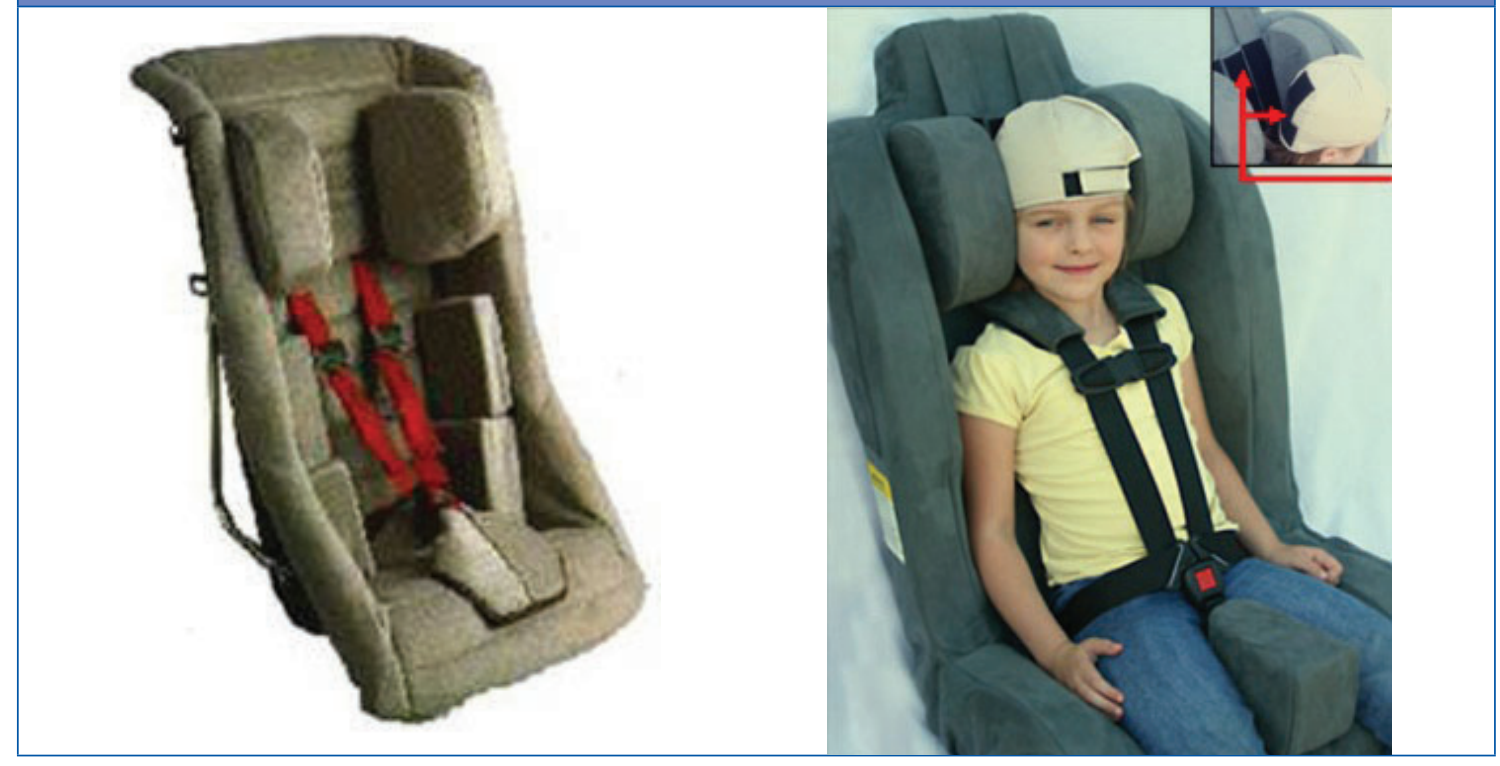

Las valoraciones anteriores de los diferentes sistemas de retención o movilidad que se indican en la Tabla 2 (solución técnica recomendada, posible o para casos especiales) resumen los consejos procedentes de otros países (principalmente EE. UU., Canadá y Suecia) y todavía no han sido analizadas en detalle en España. Por este motivo, no deben considerarse en este momento prescripciones con-

Figura 4. Asiento con arnés interno de cinco puntos para niños de hasta $38 \mathrm{~kg}$

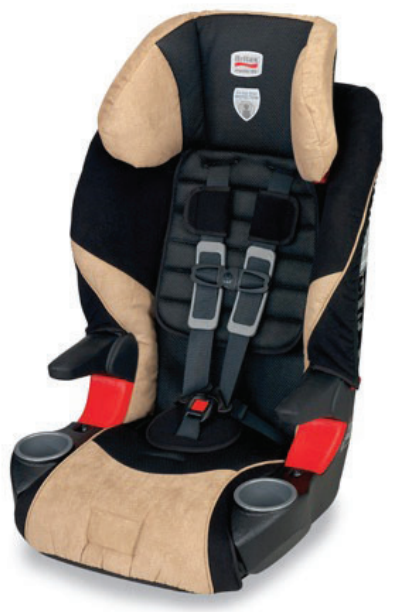

sensuadas ni, mucho menos, consejos que deban ser aplicados inmediatamente en nuestro país, sino únicamente información que aspira a ser tenida en cuenta en posteriores debates técnicos.

Además de las valoraciones incluidas en la Tabla 2 , se han encontrado los siguientes consejos generales para el transporte de niños con necesidades especiales:

Figura 5. Chaleco especial Ride Safer Travel Vest fabricado por Safe Traffic System

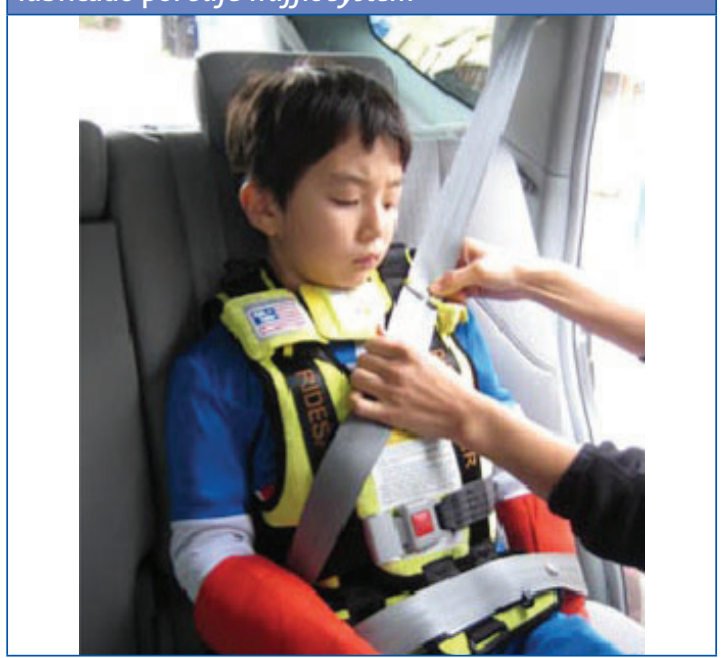




\begin{tabular}{|c|c|c|c|c|c|c|c|c|c|}
\hline & \multicolumn{8}{|c|}{ Asientos convencionales } \\
\hline & & $\begin{array}{l}\text { Cucoso } \\
\text { capazos } \\
\text { conven- } \\
\text { cionales }\end{array}$ & $\begin{array}{c}\text { Sillitas } \\
\text { para bebés } \\
\text { mirando } \\
\text { hacia atrás }\end{array}$ & $\begin{array}{l}\text { Asientos } \\
\text { convertibles } \\
\text { conven- } \\
\text { cionales } \\
\text { orientados } \\
\text { hacia atrás }\end{array}$ & $\begin{array}{c}\text { Asientos } \\
\text { convertibles } \\
\text { conven- } \\
\text { cionales } \\
\text { reclinables } \\
\text { orientados } \\
\text { hacia delante }\end{array}$ & $\begin{array}{l}\text { Asientos } \\
\text { combinados } \\
\text { conven- } \\
\text { cionales } \\
\end{array}$ & $\begin{array}{c}\text { Asientos } \\
\text { conven- } \\
\text { cionales } \\
\text { orienta- } \\
\text { dos hacia } \\
\text { delante }\end{array}$ & $\begin{array}{l}\text { Asientos } \\
\text { o cojines } \\
\text { elevadores } \\
\text { conven- } \\
\text { cionales }\end{array}$ & $\begin{array}{l}\text { Cinturón de } \\
\text { seguridad } \\
\text { para adultos } \\
\text { del vehículo } \\
\end{array}$ \\
\hline \multirow{16}{*}{ 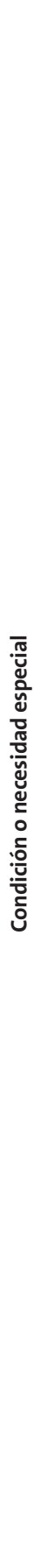 } & $\begin{array}{l}\text { 1. Bebés } \\
\text { prematuros o con } \\
\text { bajo peso al nacer }\end{array}$ & ESP & REC & POS & & & & & \\
\hline & $\begin{array}{l}\text { 2. Niños con } \\
\text { problemas } \\
\text { respiratorios }\end{array}$ & ESP & POS & & & & & & \\
\hline & $\begin{array}{l}\text { 3. Niños que } \\
\text { deban viajar } \\
\text { tumbados }\end{array}$ & REC & & & & & & & \\
\hline & $\begin{array}{l}\text { 4. Niños con } \\
\text { secuencia de Pierre } \\
\text { Robin }\end{array}$ & REC & POS & POS & & & & & \\
\hline & $\begin{array}{l}\text { 5. Niños que } \\
\text { padecen reflujo }\end{array}$ & ESP & POS & POS & & & & & \\
\hline & $\begin{array}{l}\text { 6. Niños con } \\
\text { escaso control a } \\
\text { nivel de cuello o } \\
\text { cabeza }\end{array}$ & & POS & POS & POS & POS & & POS & POS \\
\hline & $\begin{array}{l}\text { 7. Niños con } \\
\text { parálisis cerebral }\end{array}$ & & POS & POS & POS & & & & \\
\hline & $\begin{array}{l}\text { 8. Niños con } \\
\text { enfermedades } \\
\text { musculares }\end{array}$ & & REC & REC & POS & POS & POS & POS & POS \\
\hline & $\begin{array}{l}\text { 9. Niños con } \\
\text { espina bífida u } \\
\text { otras lesiones } \\
\text { medulares } \\
\text { espinales } \\
\end{array}$ & ESP & REC & REC & REC & POS & POS & & \\
\hline & $\begin{array}{l}\text { 10. Niños con } \\
\text { escoliosis }\end{array}$ & & & & & & & & \\
\hline & $\begin{array}{l}\text { 11. Niños con } \\
\text { hidrocefalia }\end{array}$ & ESP & REC & REC & REC & POS & & & \\
\hline & $\begin{array}{l}\text { 12. Niños con } \\
\text { osteogénesis } \\
\text { imperfecta ("de } \\
\text { cristal") }\end{array}$ & ESP & REC & REC & & & & & \\
\hline & $\begin{array}{l}\text { 13. Niños con } \\
\text { reumatismo }\end{array}$ & & POS & POS & POS & POS & POS & POS & POS \\
\hline & $\begin{array}{l}\text { 14. Niños con } \\
\text { acondroplasia } \\
\text { (enanismo) }\end{array}$ & & REC & REC & POS & POS & POS & POS & POS \\
\hline & $\begin{array}{l}\text { 15. Niños grandes } \\
\text { u obesos }\end{array}$ & & POS & POS & POS & POS & POS & POS & POS \\
\hline & $\begin{array}{l}\text { 16. Niños que } \\
\text { usan gafas }\end{array}$ & & POS & POS & POS & POS & POS & POS & POS \\
\hline
\end{tabular}


Tabla 2a. Resumen de condiciones especiales y soluciones técnicas disponibles (asientos convencionales) (cont.)

\begin{tabular}{|c|c|c|c|c|c|c|c|c|c|}
\hline & \multicolumn{8}{|c|}{ Asientos convencionales } \\
\hline & & \multirow[t]{2}{*}{$\begin{array}{l}\text { Cucos o } \\
\text { capazos } \\
\text { conven- } \\
\text { cionales }\end{array}$} & \multirow{2}{*}{$\begin{array}{c}\begin{array}{c}\text { Sillitas } \\
\text { para bebés } \\
\text { mirando } \\
\text { hacia atrás }\end{array} \\
\text { POS }\end{array}$} & \multirow{2}{*}{$\begin{array}{c}\begin{array}{c}\text { Asientos } \\
\text { convertibles } \\
\text { conven- } \\
\text { cionales } \\
\text { orientados } \\
\text { hacia atrás }\end{array} \\
\text { POS }\end{array}$} & \multirow{2}{*}{\begin{tabular}{|c|}
$\begin{array}{c}\text { Asientos } \\
\text { convertibles } \\
\text { conven- } \\
\text { cionales } \\
\text { reclinables } \\
\text { orientados } \\
\text { hacia delante }\end{array}$ \\
\\
POS
\end{tabular}} & \multirow{2}{*}{$\begin{array}{c}\begin{array}{c}\text { Asientos } \\
\text { combinados } \\
\text { conven- } \\
\text { cionales }\end{array} \\
\text { POS }\end{array}$} & \multirow{2}{*}{$\begin{array}{c}\text { Asientos } \\
\text { conven- } \\
\text { cionales } \\
\text { orienta- } \\
\text { dos hacia } \\
\text { delante }\end{array}$} & \multirow{2}{*}{$\begin{array}{l}\text { Asientos } \\
\text { o cojines } \\
\text { elevadores } \\
\text { conven- } \\
\text { cionales } \\
\text { POS }\end{array}$} & \multirow{2}{*}{$\begin{array}{c}\begin{array}{c}\text { Cinturón de } \\
\text { seguridad } \\
\text { para adultos } \\
\text { del vehículo }\end{array} \\
\text { POS }\end{array}$} \\
\hline \multirow{12}{*}{ 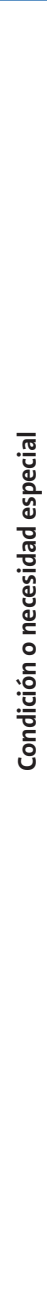 } & $\begin{array}{l}\text { 17. Niños con } \\
\text { problemas de } \\
\text { visión }\end{array}$ & & & & & & & & \\
\hline & $\begin{array}{l}\text { 18. Niños con } \\
\text { problemas } \\
\text { auditivos }\end{array}$ & & POS & POS & POS & POS & POS & POS & POS \\
\hline & $\begin{array}{l}\text { 19. Niños con } \\
\text { vendajes }\end{array}$ & & POS & POS & POS & POS & POS & POS & POS \\
\hline & $\begin{array}{l}\text { 20. Niños con } \\
\text { collarines }\end{array}$ & & POS & POS & POS & POS & POS & POS & POS \\
\hline & $\begin{array}{l}\text { 21. Niños con } \\
\text { traqueotomías }\end{array}$ & & REC & REC & POS & POS & POS & POS & POS \\
\hline & $\begin{array}{l}\text { 22. Niños } \\
\text { con tubos de } \\
\text { alimentación o } \\
\text { gastrointestinales }\end{array}$ & & POS & POS & POS & POS & POS & POS & POS \\
\hline & $\begin{array}{l}\text { 23. Niños con } \\
\text { yesos }\end{array}$ & ESP & POS & POS & POS & POS & POS & POS & POS \\
\hline & $\begin{array}{l}\text { 24. Niños } \\
\text { portadores de halo } \\
\text { craneal }\end{array}$ & & REC & REC & & & & & \\
\hline & $\begin{array}{l}\text { 25. Niños con } \\
\text { comportamientos } \\
\text { difíciles }\end{array}$ & & POS & POS & POS & POS & POS & POS & POS \\
\hline & $\begin{array}{l}\text { 26. Niños con } \\
\text { retrasos en el } \\
\text { desarrollo }\end{array}$ & & REC & REC & POS & POS & & & \\
\hline & $\begin{array}{l}\text { 27. Niños que se } \\
\text { desplazan en sillas } \\
\text { de ruedas }\end{array}$ & & & & & & & & \\
\hline & $\begin{array}{l}\text { 28. Niños que } \\
\text { deben viajar con } \\
\text { equipo médico }\end{array}$ & & & & & & & & \\
\hline
\end{tabular}

REC: recomendado; POS: posible; ESP: especial.

- Las exenciones al uso de sistemas de retención deberían ser realmente excepcionales. Al igual que el resto de niños, los niños con necesidades especiales deberían viajar correctamente sujetos en un dispositivo de retención adecuado para su edad, peso y condición médica particular.

- Los asientos infantiles orientados hacia atrás son más seguros para cualquier niño, pero es- pecialmente para muchos niños con necesidades especiales. Deberían ser la opción preferida o recomendada en la mayoría de los casos.

- Cuando el niño viaje en un asiento convertible (aquel que puede instalarse tanto mirando hacia atrás, para niños pequeños, como hacia delante, para niños mayores) mirando hacia delante o en un asiento convencional también orientado hacia delante, conviene seleccionar 
Tabla 2b. Resumen de condiciones especiales y soluciones técnicas disponibles (asientos especializados)

\begin{tabular}{|c|c|c|c|c|c|c|c|c|c|c|c|c|}
\hline & \multicolumn{11}{|c|}{ Asientos convencionales } \\
\hline & & $\begin{array}{l}\text { Asientos } \\
\text { conver- } \\
\text { tibles } \\
\text { especia- } \\
\text { lizados }\end{array}$ & \begin{tabular}{|l} 
Asientos \\
especia- \\
les para \\
niños con \\
yesos en \\
la cadera
\end{tabular} & $\begin{array}{l}\text { Chalecos } \\
\text { de seguri- } \\
\text { dad para } \\
\text { niños } \\
\text { tipo E-Z- } \\
\text { On }\end{array}$ & \begin{tabular}{|c|} 
Chalecos \\
modificados \\
para niños \\
que deben \\
viajar tum- \\
bados
\end{tabular} & \begin{tabular}{|c|} 
Asientos \\
médicos \\
orien- \\
tados \\
hacia \\
delante
\end{tabular} & $\begin{array}{l}\text { Sillas de } \\
\text { ruedas } \\
\text { (siste- } \\
\text { mas de } \\
\text { movili- } \\
\text { dad) }\end{array}$ & $\begin{array}{c}\text { Cintu- } \\
\text { rones o } \\
\text { chalecos } \\
\text { de apoyo } \\
\text { postural }\end{array}$ & $\begin{array}{l}\text { Colla- } \\
\text { rines } \\
\text { flexi- } \\
\text { bles }\end{array}$ & $\begin{array}{c}\text { Toallas } \\
\text { enrolladas } \\
\text { o tubos de } \\
\text { espuma de } \\
\text { apoyo postu- } \\
\text { ral adicional }\end{array}$ & $\begin{array}{l}\text { Equi- } \\
\text { po } \\
\text { mé- } \\
\text { dico }\end{array}$ & $\begin{array}{l}\text { Vehícu- } \\
\text { los } \\
\text { adap- } \\
\text { tados }\end{array}$ \\
\hline & $\begin{array}{l}\text { 1. Bebés } \\
\text { prematuros o } \\
\text { con bajo peso } \\
\text { al nacer }\end{array}$ & & & & & & & & & ESP & & \\
\hline & $\begin{array}{l}\text { 2. Niños con } \\
\text { problemas } \\
\text { respiratorios }\end{array}$ & & & & & & & & & & & \\
\hline & $\begin{array}{l}\text { 3. Niños que } \\
\text { deban viajar } \\
\text { tumbados }\end{array}$ & & & & ESP & & & & & & & \\
\hline & $\begin{array}{l}\text { 4. Niños con } \\
\text { secuencia de } \\
\text { Pierre Robin } \\
\end{array}$ & & & & & & & & & & & \\
\hline & $\begin{array}{l}\text { 5. Niños que } \\
\text { padecen } \\
\text { reflujo }\end{array}$ & & & & ESP & & & & & & & \\
\hline$\overline{\Xi \Xi}$ & \begin{tabular}{|l|} 
6. Niños \\
con escaso \\
control a nivel \\
de cuello o \\
cabeza \\
\end{tabular} & & & ESP & & ESP & & & & ESP & & \\
\hline$\frac{\overrightarrow{0}}{\bar{d}}$ & $\begin{array}{l}\text { 7. Niños } \\
\text { con parálisis } \\
\text { cerebral }\end{array}$ & & ESP & ESP & & ESP & ESP & ESP & ESP & ESP & & \\
\hline 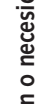 & $\begin{array}{l}\text { 8. Niños con } \\
\text { enfermedades } \\
\text { musculares }\end{array}$ & & & & & & & ESP & ESP & & & \\
\hline $\begin{array}{l}\frac{\overline{0}}{\overline{0}} \\
\frac{0}{\overline{0}} \\
\overline{0}\end{array}$ & \begin{tabular}{|l|} 
9. Niños con \\
espina bífida u \\
otras lesiones \\
medulares \\
espinales \\
\end{tabular} & & & & & ESP & ESP & ESP & & & & \\
\hline & $\begin{array}{l}\text { 10. Niños con } \\
\text { escoliosis }\end{array}$ & & & & ESP & ESP & ESP & & & & & \\
\hline & $\begin{array}{l}\text { 11. Niños con } \\
\text { hidrocefalia }\end{array}$ & & & & & ESP & & & & & & \\
\hline & $\begin{array}{l}\text { 12. Niños con } \\
\text { osteogénesis } \\
\text { imperfecta } \\
\text { ("de cristal") }\end{array}$ & & ESP & & & & ESP & ESP & & & & \\
\hline & $\begin{array}{l}\text { 13. Niños con } \\
\text { reumatismo }\end{array}$ & & & & & & & & ESP & ESP & & \\
\hline & $\begin{array}{l}\text { 14. Niños con } \\
\text { acondroplasia } \\
\text { (enanismo) }\end{array}$ & & & & & ESP & & & & & & \\
\hline & $\begin{array}{l}\text { 15. Niños } \\
\text { grandes u } \\
\text { obesos }\end{array}$ & ESP & & & & ESP & & & & & & \\
\hline & $\begin{array}{l}\text { 16. Niños que } \\
\text { usan gafas }\end{array}$ & & & & & & & & & & & \\
\hline
\end{tabular}


Tabla 2b. Resumen de condiciones especiales y soluciones técnicas disponibles (asientos especializados) (cont.)

\begin{tabular}{|c|c|c|c|c|c|c|c|c|c|c|c|c|}
\hline & \multicolumn{11}{|c|}{ Asientos convencionales } \\
\hline & & $\begin{array}{l}\text { Asientos } \\
\text { conver- } \\
\text { tibles } \\
\text { especia- } \\
\text { lizados }\end{array}$ & $\begin{array}{l}\text { Asientos } \\
\text { especia- } \\
\text { les para } \\
\text { niños con } \\
\text { yesos en } \\
\text { la cadera }\end{array}$ & $\begin{array}{l}\text { Chalecos } \\
\text { de seguri- } \\
\text { dad para } \\
\text { niños } \\
\text { tipo E-Z- } \\
\text { On }\end{array}$ & $\begin{array}{l}\text { Chalecos } \\
\text { modificados } \\
\text { para niños } \\
\text { que deben } \\
\text { viajar tum- } \\
\text { bados }\end{array}$ & $\begin{array}{c}\text { Asientos } \\
\text { médicos } \\
\text { orien- } \\
\text { tados } \\
\text { hacia } \\
\text { delante }\end{array}$ & $\begin{array}{l}\text { Sillas de } \\
\text { ruedas } \\
\text { (siste- } \\
\text { mas de } \\
\text { movili- } \\
\text { dad) }\end{array}$ & $\begin{array}{l}\text { Cintu- } \\
\text { rones o } \\
\text { chalecos } \\
\text { de apoyo } \\
\text { postural }\end{array}$ & $\begin{array}{l}\text { Colla- } \\
\text { rines } \\
\text { flexi- } \\
\text { bles }\end{array}$ & $\begin{array}{c}\text { Toallas } \\
\text { enrolladas } \\
\text { o tubos de } \\
\text { espuma de } \\
\text { apoyo postu- } \\
\text { ral adicional }\end{array}$ & $\begin{array}{l}\text { Equi- } \\
\text { po } \\
\text { mé- } \\
\text { dico }\end{array}$ & $\begin{array}{l}\text { Vehi- } \\
\text { culos } \\
\text { adap- } \\
\text { tados }\end{array}$ \\
\hline & $\begin{array}{l}\text { 17. Niños con } \\
\text { problemas de } \\
\text { visión }\end{array}$ & & & & & & & & & & & \\
\hline & $\begin{array}{l}\text { 18. Niños con } \\
\text { problemas } \\
\text { auditivos }\end{array}$ & & & & & & & & & & & \\
\hline & $\begin{array}{l}\text { 19. Niños con } \\
\text { vendajes }\end{array}$ & & & & & & & & & & & \\
\hline & $\begin{array}{l}\text { 20. Niños con } \\
\text { collarines }\end{array}$ & & & & & & & & REC & & & \\
\hline & $\begin{array}{l}\text { 21. Niños con } \\
\text { traqueotomías }\end{array}$ & & & & & & & & & & & \\
\hline 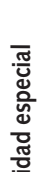 & $\begin{array}{l}\text { 22. Niños } \\
\text { con tubos de } \\
\text { alimentación o } \\
\text { gastrointesti- } \\
\text { nales }\end{array}$ & & & & & & & & & & & \\
\hline 芯 & $\begin{array}{l}\text { 23. Niños con } \\
\text { yesos }\end{array}$ & ESP & ESP & & & ESP & & & & ESP & & \\
\hline$\frac{\overline{0}}{\mathrm{u}}$ & $\begin{array}{l}\text { 24. Niños } \\
\text { portadores de } \\
\text { halo craneal }\end{array}$ & & & & ESP & & & & & & & \\
\hline & $\begin{array}{l}\text { 25. Niños con } \\
\text { comportami- } \\
\text { entos difíciles }\end{array}$ & & & ESP & & ESP & & & & & & \\
\hline & $\begin{array}{l}\text { 26. Niños con } \\
\text { retrasos en el } \\
\text { desarrollo }\end{array}$ & & & ESP & ESP & ESP & ESP & & & & & \\
\hline & $\begin{array}{l}\text { 27. Niños que } \\
\text { se desplazan } \\
\text { en sillas de } \\
\text { ruedas }\end{array}$ & & & & & & REC & & & & & ESP \\
\hline & $\begin{array}{l}\text { 28. Niños que } \\
\text { deben viajar } \\
\text { con equipo } \\
\text { médico }\end{array}$ & & & & & & & & & & REC & ESP \\
\hline
\end{tabular}

REC: recomendado; POS: posible; ESP: especial.

un asiento cuyo respaldo pueda ser ligeramente reclinado, lo que suele mejorar el apoyo para la parte superior del cuerpo del niño y la comodidad.

- En el caso de muchas condiciones médicas -como niños pequeños, niños con traqueotomías o niños con gafas-, no se recomiendan los asientos infantiles que dispongan, como parte de su mecanismo de retención, de bandejas o escudos situados delante del pecho o la cabeza del niño. En caso de frenazo o colisión, el niño podría golpear dichos elementos.

- Siempre que sea posible, resulta más seguro transferir a los niños que usen sillas de ruedas 
desde estas a un sistema de retención infantil que vaya firmemente instalado en el vehículo. La silla de ruedas tiene que ser posteriormente asegurada en el maletero o suelo del vehículo.

- El equipo médico que deba viajar con el niño tiene que ir siempre asegurado al vehículo para evitar que se convierta en un proyectil e impacte contra los ocupantes en caso de accidente. Si dicho equipo necesita energía eléctrica durante el transporte, la duración de las baterías tiene que superar el doble de la duración estimada del viaje.

- Nunca debe transportarse a ningún niño o pasajero, y por supuesto a ningún niño con necesidades especiales (como un niño con yeso), tumbado en un asiento del vehículo al que se le haya reclinado el respaldo. En caso de accidente, el niño no estaría sujeto y podrían producirse lesiones con el cinturón de seguridad del vehículo $u$ otros elementos del interior de este.

- En caso de no poder trasladar al niño en ninguno de los tipos de sistemas de retención de la Tabla 2, la única alternativa segura sería el transporte en ambulancia.

\section{Otros resultados}

Como parte de este trabajo, también se realizó un sondeo de carácter exploratorio en ortopedias y empresas especializadas en productos de rehabilitación en España. En concreto, se contactó con las siguientes entidades (por orden alfabético): Aparells Ortopedics Curto, HIDELASA, Mundo Dependencia, Ortoibérica, Ortopedia Arrahona, Ortopedia BCN, Reha Girona y Rehatrans. Como resultado de dicho sondeo, se han identificado los siguientes asientos infantiles especialmente indicados para niños con necesidades especiales que están disponibles en España (las siguientes referencias a productos comerciales se ofrecen únicamente a título informativo y no implican ningún tipo de valoración o recomendación) (Tabla 3).

La anterior información fue complementada con un segundo sondeo entre los fabricantes internacionales de sistemas de retención para niños con necesidades especiales que habían sido identifica- dos en la revisión de la bibliografía internacional. Bien a través de sus formularios de contacto online, bien a través de las direcciones de correo electrónico que aparecían en sus páginas de Internet, se intentó contactar con los siguientes fabricantes o distribuidores (todos ellos estadounidenses, excepto en los casos en los que se indica entre paréntesis el país del fabricante o distribuidor): Angel Guard, BESI Inc, Britax, Carfix (Dinamarca), Child Source, Columbia Medical, EZ-On, JCM Seating, Medifix Services Limited, LifeTec Queensland (Australia), Merritt Manufacturing, Q'Vest, Recaro EE. UU., Recaro (Reino Unido), Reha Partner (Alemania), Safe Traffic System, Sammons Preston, Snug Seat, Special Tomato, Sunrise Medical y Tumble Forms. Los fabricantes Merritt Manufacturing, Safe Traffic System y Snug Seat fueron los únicos que respondieron, indicando en todos los casos que sus productos no están homologados según la normativa europea ECE R44/04, por lo que no es posible su uso en Europa ni se distribuyen en nuestro continente.

\section{DISCUSIÓN}

Tras la revisión de la bibliografía internacional, puede concluirse que EE. UU. es, sin ningún tipo de duda, el país que más atención ha prestado a los niños con necesidades especiales en el automóvil. Seguramente debido a ello, es también el país donde el mercado ofrece una mayor variedad de productos o soluciones técnicas.

Con objeto de aportar elementos para el debate, este artículo y el trabajo en el que está basado han revisado la bibliografía internacional relativa a los niños con necesidades especiales y su seguridad en el automóvil. El trabajo puede considerarse una de las obras más completas sobre esta materia específica a nivel internacional, ya que resume y procesa la información identificada en fuentes principalmente estadounidenses, canadienses, suecas, etc. Los padres y los tutores de niños con necesidades especiales deberían ser aconsejados por los profesionales médicos (pediatras, terapeutas ocupacio- 
Tabla 3. Resumen de soluciones técnicas disponibles en España

\begin{tabular}{|c|c|c|c|c|}
\hline Producto & Características básicas & Distribuidor & $\begin{array}{l}\text { Precio } \\
\text { aprox. }\end{array}$ & Imagen \\
\hline Monterey & $\begin{array}{l}\text { Silla de los grupos I a III (de } 9 \text { a } 36 \mathrm{~kg} \\
\text { de peso), con arnés de } 5 \text { puntos (grupo } \\
\text { I), soporte lumbar ajustable en altura, } \\
\text { respaldo ajustable en altura, cumple con } \\
\text { normativa ECE R44/03 }\end{array}$ & $\begin{array}{l}\text { HIDELASA, Mundo } \\
\text { Dependencia, Reha } \\
\text { Girona }\end{array}$ & 650 & \\
\hline Recaro Start & $\begin{array}{l}\text { Silla de los grupos I a III (de } 9 \text { a } 36 \mathrm{~kg} \\
\text { de peso), con arnés de } 5 \text { puntos (grupo } \\
\text { I), altura de respaldo y profundidad de } \\
\text { asiento regulable, asiento basculante, } \\
\text { arnés de } 5 \text { puntos, soportes laterales de } \\
\text { cabeza, varios accesorios }\end{array}$ & $\begin{array}{l}\text { HIDELASA, Mundo } \\
\text { Dependencia }\end{array}$ & 940 & \\
\hline Recaro Monza & $\begin{array}{l}\text { ECE R 44/04 grupos II y III (15-36 } \\
\mathrm{kg} \text { ), base giratoria, arnés de } 5 \text { puntos, } \\
\text { sistema integrado de circulación de aire, } \\
\text { reposacabezas y soportes de tronco, } \\
\text { amplia variedad de accesorios }\end{array}$ & Reha Girona & & \\
\hline Recaro Sport & $\begin{array}{l}\text { ECE R 44/04 grupos I-II-III (9-36 kg), } \\
\text { base giratoria, basculación de asiento, } \\
\text { acolchado reductor de asiento, arnés de } 5 \\
\text { puntos con ajuste central, reposacabezas }\end{array}$ & Reha Girona & & \\
\hline $\begin{array}{l}\text { Monterey con } \\
\text { base SAM }\end{array}$ & $\begin{array}{l}\text { Silla de los grupos I a III, cumple con ECE } \\
\text { R44/04, cinturón de } 5 \text { puntos confort, } \\
\text { base SAM giratoria y deslizante del } \\
\text { asiento, silla basculante, reposacabezas } \\
\text { y soporte lumbar ajustable en altura, } \\
\text { reposapiés regulable en altura }\end{array}$ & Reha Girona & & \\
\hline Q'Vest & $\begin{array}{l}\text { Cinturón postural (sistema de sujeción } \\
\text { adicional que no reemplaza al cinturón } \\
\text { de seguridad o sistema de retención } \\
\text { homologado). Desde } 9 \text { hasta } 68 \mathrm{~kg} \text { de } \\
\text { peso. Sin instalación especial }\end{array}$ & Rehatrans & 125 & \\
\hline Careva & $\begin{array}{l}\text { Cinturón postural (sistema de sujeción } \\
\text { adicional que no reemplaza al cinturón } \\
\text { de seguridad o sistema de retención } \\
\text { homologado). Se puede instalar en todo } \\
\text { tipo de asientos que tengan apertura } \\
\text { entre la base del asiento y el respaldo. } \\
\text { Para ocupantes de hasta } 75 \mathrm{~kg} \text { de peso }\end{array}$ & Rehatrans & 510 & \\
\hline Carony Kids & $\begin{array}{l}\text { Sistema de movilidad (silla de ruedas) } \\
\text { cuyo asiento se acopla al vehículo, siendo } \\
\text { el mismo asiento de la silla de ruedas el } \\
\text { del vehículo. Cojín ajustable en el cuello } \\
\text { y zona lumbar. Evita tener que levantar } \\
\text { al niño del asiento para sentarlo en el } \\
\text { vehículo. Requiere el uso de los cinturones } \\
\text { de seguridad del vehículo }\end{array}$ & Rehatrans & 3975 & \\
\hline
\end{tabular}


nales, etc.) sobre las alternativas de movilidad segura de sus hijos o niños, incluidos los desplazamientos en el vehículo particular. Los profesionales médicos deberían disponer de toda la información precisa para dicho asesoramiento. De hecho, antes de que los padres o tutores tomen cualquier decisión, es necesario que analicen con el pediatra del niño cuáles son sus necesidades específicas a la hora del transporte, por ejemplo, cuáles son las necesidades posturales concretas.

Los asientos orientados hacia atrás, más seguros para cualquier niño, están especialmente recomendados para muchas de las categorías de niños con necesidades especiales (como, por ejemplo, los "niños de cristal"), por lo que sería muy deseable aumentar el número de asientos orientados hacia atrás del grupo I (de 9 a $18 \mathrm{~kg}$ de peso) disponibles en España y lanzar una campaña general de concienciación sobre la mayor protección que ofrecen los asientos orientados hacia atrás.

Cuando un niño con necesidad de cuidados médicos especiales abandona el hospital, el protocolo médico de alta debería incluir información y formación sobre el uso del sistema de retención más adecuado en cada caso. En caso necesario, podría incluir considerarse, como sucede en otros países, que dicho protocolo incluya la entrega o cesión a los padres o cuidadores de un sistema de retención infantil adecuado.

Los asientos específicos para niños con necesidades especiales son varias veces más caros que los asientos infantiles convencionales. Por si esto fuera poco, en ciertas ocasiones, las necesidades especiales son temporales o de corta duración. Por estos motivos, se deberían promover los programas de subvención, préstamo u otros modos de proporcionar asientos infantiles de seguridad durante periodos cortos de tiempo. Con ello se facilitarían las soluciones seguras de movilidad en el automóvil durante el periodo de discapacidad y a un coste aceptable. Esto ayudaría a evitar que los padres, como puede suceder en la práctica, ingenien soluciones caseras (y peligrosas) para mantener un cierto grado de autonomía y movilidad familiar.
En España son todavía pocos los modelos de asientos para niños con necesidades especiales a la venta. Sería conveniente examinar en detalle qué otros modelos o tipos de asientos deberían ofrecerse en el mercado español.

Los fabricantes de asientos infantiles, los legisladores, los profesionales de la salud y la rehabilitación, así como también las asociaciones de consumidores, deberían, todos ellos, colaborar para mejorar la oferta y el acceso a soluciones seguras para el transporte en el automóvil de los niños con necesidades especiales, y a un coste razonable.

Este artículo tabula y clasifica los diversos tipos de soluciones técnicas para alrededor de una treintena de categorías de necesidades especiales. Dichos consejos y soluciones técnicas proceden a menudo de Norteamérica y es necesario que sean validados por la comunidad médica española. En concreto, deberían analizarse, una a una, las soluciones o alternativas de movilidad utilizadas en otros países y que han sido incluidas en este trabajo, y debería decidirse cuáles son relevantes, interesantes o necesarias en España. El resultado final de este debate debería ser un documento consensuado por la comunidad de Pediatría española con recomendaciones sobre la materia.

\section{CONCLUSIONES}

En el ámbito que nos ocupa, los niños con necesidades especiales son todos aquellos niños con algún tipo de condición médica particular que exija unos cuidados o una atención especial a la hora de su transporte. Entre dichas condiciones (la revisión internacional de manuales y guías refiere aproximadamente 30 condiciones distintas) se incluyen, por ejemplo, los niños prematuros o bajos de peso al nacer, los niños con espina bífida, con parálisis cerebral, con enfermedades neuromusculares o con falta de control muscular, con huesos de cristal, con autismo $u$ otros desórdenes de comportamiento, etc.

El número de niños con necesidades especiales en España se ha estimado en un mínimo de 60000 
niños. Por lo general, en España existe muy poca información específica sobre seguridad en el automóvil y niños con necesidades especiales. En otros países como EE. UU., Canadá o Suecia, los padres y los profesionales médicos disponen de manuales o guías de referencia sobre la temática. Por otra parte, el mercado de soluciones técnicas para el transporte seguro en el automóvil de niños con necesidades especiales es muy limitado en España.

La principal conclusión de este artículo es que resulta necesario abrir un debate para que la comunidad médica española adopte sus propias recomendaciones para el transporte seguro de los niños con necesidades especiales.

Como parte de dicho debate, se debería analizar cuáles de los asientos o soluciones técnicas que están disponibles en otros países también son relevantes en España, con el objetivo final de que también estén disponibles en nuestro país.

En el caso de aquellos niños con necesidades especiales permanentes o crónicas, se debería habilitar un programa de ayudas económicas a la adquisición de las soluciones técnicas adecuadas. Algunas sillas de seguridad para niños con necesidades especiales cuestan más de 1000 euros, por ejemplo, y dicho precio no incluye ajustes o modificaciones personalizadas.

\section{BIBLIOGRAFÍA}

1. Monclús J. Panorama de la seguridad infantil en el automóvil (años 1990-2010). 4.e ed. Trabajo realizado con la colaboración y supervisión de la Fundación Mapfre, 2012 [en línea] [consultado el 13/01/2013]. Disponible en www.mapfre.com/ccm/content/do cumentos/fundacion/seg-vial/investigacion/20anios-de-asientos-infantiles.pdf

2. Observatorio Nacional de Seguridad Vial. Indicadores de uso de sistemas de seguridad y teléfono móvil 2010. Madrid: Dirección General de Tráfico (DGT), Ministerio del Interior; 2011 [en línea] [consultado el 17/01/2013]. Disponible en www.dgt.es/portal/es/
En el caso de niños con necesidades especiales temporales, sería oportuno desarrollar un programa de préstamo o alquiler de corto plazo de las citadas ayudas técnicas. El programa debería reducir los costes asociados a dichos dispositivos y, sobre todo, el tiempo necesario para acceder a los mismos (en el peor de los casos, la entrega de determinados asientos especiales puede requerir un plazo de tiempo superior a la duración de la necesidad especial temporal).

Sería necesario establecer un mecanismo para poner al alcance de los profesionales médicos, los padres y los tutores información especializada sobre el transporte seguro de los niños con necesidades especiales.

\section{CONFLICTO DE INTERESES}

El estudio en el que está basado este trabajo ha sido financiado por la Fundación MAPFRE.

Los autores declaran no presentar conflictos de intereses en relación con la preparación y publicación de este artículo.

\section{ABREVIATURAS}

INE: Instituto Nacional de Estadística.

seguridad_vial/estudios_informes/estudios_infor mes157.htm

3. FIA, Foundation for the Automobile and Society. Cinturones de seguridad y sistemas de retención infantil: un manual de seguridad vial para decisores y profesionales. Londres: FIA Foundation for the Automobile and Society; 2009 [en línea] [consultado el 13/01/2013]. Disponible en http://whqlibdoc.who. int/road_safety/2009/9780956140333_spa.pdf

4. Monclús J. Los niños con necesidades especiales y su seguridad en el automóvil. Trabajo realizado con la colaboración y supervisión de la Fundación MAPFRE, 2012. Pendiente de publicación.

5. Bull M, Agran P, Laraque D, Pollack SH, Smith GA, Spivak HR, et al. American Academy of Pediatrics. Committee on Injury and Poison Prevention. Transporting 
children with special health care needs. Pediatrics. 1999;104:988-92.

6. American Academy of Pediatrics. Safe Transportation of Children With Special Needs: A Guide for Families. American Academy of Pediatrics; 2002.

7. Transport Canada. Transporting Infants and Children with Special Needs in Personal Vehicles - A Best Practices Guide for Healthcare Practitioners. Road Safety, Transport Canada, 2008 [en línea] [consultado el 13/01/2013]. Disponible en www.tc.gc.ca/me dia/documents/roadsafety/TP14772e.pdf

8. Falkmer T, Paulsoon K. Safe Ride - Traffic safety for children with disabilities. National Association for Disabled Children \& Youth (RBU), Swedish National Road \& Transport Research Institute (Statens vägoch transportforskningsinstitut - VTI), Swedish Road Administration and Swedish Institute of Assistive Technology; 2003 [en línea] [consultado el 13/01/2013]. Disponi- ble en www.vti.se/Global/Om\%20VTI/\%c3\%85ka\%20

s\%c3\%a4kert_eng.pdf?epslanguage=en

9. Goodwill Industries. Child Passenger Safety Handbook - handbook for children with special needs. Goodwill, Easter Seals Miami Valley, 2008 [en línea] [consultado el 13/01/2013]. Disponible en: http:// www.gesmv.org/gesmv/assets/File/Car_seat_hand book_2010_final_web.pdf

10. Safe Ride News Publications. Child Restraints for Children with Special Needs-A Healthcare Provider's Guide. FACT SHEET. Safe Ride News Pub. D2 Revised 1/2008.

11. Anund A, Falkmer T, Forsman A, Gustafsson S, Matsotms $Y$, Sörensen G, et al. Child safety in cars - Literature review. Swedish National Road and Transport Research Institute (Statens vägoch transportforskningsinstitut - VTI). VTI Rapport 489å; 2003 [en línea] [consultado el 13/01/2013]. Disponible en: www.vti.se/EPiBrowser/Publikationer/R489A.pdf 